\title{
The Use of the TMCS Measurement and Control Simulator on the Ground Simulation Experiment of the Rotary-steering Dilling Measurement System
}

\author{
Xiliang ZHANG \\ CNOOC EnerTech - Drilling \& Production Co. Tianjin, China \\ Ruirong DANG \\ $X i$ 'an Shiyou University, Xi'an, China \\ Renqi MA, Fenghui ZHANG \\ CNOOC EnerTech - Drilling \& Production Co. Tianjin 300451, China
}

\begin{abstract}
This paper introduces in detail the TMCS simulator in the Measurement while drilling system of rotary steering drilling tool in ground simulation Experiment system. Focusing on of TMCS measurement and control simulator, and the realization of steering drilling measurement simulation test system principle of work process
\end{abstract}

KEYWORD: Steering drilling; TMCS measurement and control simulator; Measurement system of steering drilling; Ground simulation Experiment

\section{INTRODUCTION}

The Rotary Steering Drilling System at the time of the drill string rotary drilling, with a directional drilling function in real time steering drilling system. The measurement system of steering drilling is a rotary steerable drilling steerable drilling system to achieve "precise control" the core part of the drilling process to provide wellhead inclination, azimuth, and other real-time measurement of surface drilling data. Best drill internal electronic measuring tool will automatically adjust the position of the drill, make sure the reservoir and the wellbore to accurately hit the walk through the favor of oil and gas exploration; According to the information obtained, may issue instructions from the wellhead to maintain or change the drill track location.

To verify the measurement system of steering drilling stability and accuracy in rotary steering drilling begins to go down a job before, the system needs to be fully ground simulation tests to ensure steerable drilling measurement system is working properly. TMCS measurement and control simulator is actually a measurement and control unit contains gravity accelerometer, a microprocessor and associated circuitry. It can detect a variety of signal reception. get the right data through a certain algorithm. The most commonly used measurement module verification steering drilling down-hole measurements oblique rotation state control and accuracy, is a way to change the attitude of the test bench space through the design, driven rotary steering drilling measurement module simulation drill collar to specify the speed of rotation, the test system real-time angle, speed, control axis angle, torque and the inclination of the test bench and other parameters measured, processed and stored and analyzed.

This method can measure hardware and software of steering drilling system modules to conduct a comprehensive testing and validation, but having a apparent different the dynamic process of the rotary drilling rig and the rotation of the test bench.

This article describes using high dynamic TMCS measurement and control simulator, motion information down-hole with drilling tools based on pre-set or a combination of real-time simulation, real and effective rotary steering drilling tools to simulate three-dimensional motion attitude, can achieve a comprehensive validation of the steering drilling measurement system. The following detailed description of the system.

\section{TMCS MEASUREMENT AND CONTROL SIMULATOR PROFILE}

TMCS measurement and control simulator can drill assembly drilling dynamic environment, produce accurate installation on steering drilling received inclination measurement system based on down-hole rotary steerable, azimuth and tool face angle signal can be used to guide drilling measurement system functional test, measurement accuracy assessment, 
target motion characteristics simulation. Selected TMCS measurement and control simulator consists of pre-positioning data steerable drilling threedimensional trajectory, WOB and torque drilling parameters such as generators and computer subsystems.

The drilling parameter produce a variety of mixed uses a dedicated data signal modulation scheme, which can effectively reduce the direct digital synthesis technology brought drift, dislocation and loss. Control process shown in Figure 1.

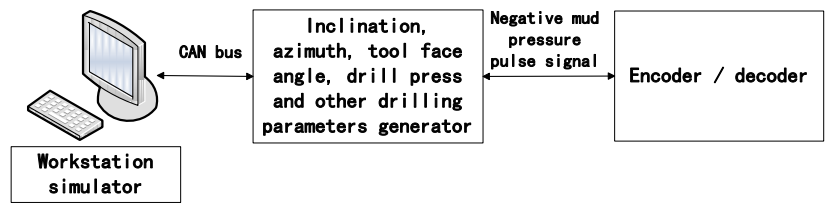

Figure 1. TMCS control process

On the workstation simulator programming movement characteristics of rotary steering drilling assembly can also be preset orbit stored in document form workstation simulator, the simulator will be run in accordance with the information stored threedimensional simulation of down-hole drilling trajectory.

\section{SIMULATED TEST STEERING DRILLING SYSTEM COMPONENTS AND WORKING PRINCIPLE}

The ground simulation experiment for in steering drilling measurement system, that components shown in Figure 2. The simulation system works as follows.

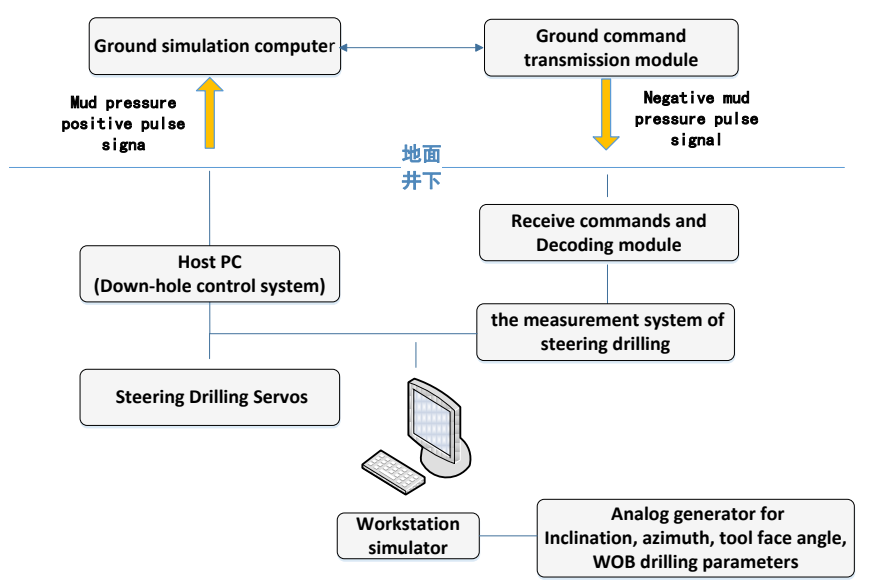

Figure 2. Schematic diagram of steering drilling measurement system simulation experiment system

Steering drilling system collects the real-time inclination, azimuth and tool face angle based on the output of the sensor data with from measurement data model of the workstation simulator, and analog outputs sent to the host PC (the down-hole control center), Which of the control software to collect data based on the real-time input wellbore trajectory calculation, measurement and steering drilling system solver of inclination, azimuth and tool face angle information were to do the filtering. Then inclination, azimuth and tool face angle deviation with the output of the state variable filter, will feedback to steering drilling measurement system solver error correction, ground simulation computer real-time acquisition host computer (the underground control center) of the D / A output, in a way to get uploading these amendments of the host computer (down-hole control center) by the downhole mud pressure pulse signal, and according to the mathematical model of the D / A output and a steering servo mechanism, solving actual servo movement at this time, feeding rotary steering drilling of BHA model for controlling motion to calculate the angular velocity of the solution, attitude, position, velocity and acceleration information, converted into real-time drilling parameters which ground engineers need , and to be ground control instructions that were encoded for transferring by mud pressure module negative pulse signals. To be received with decoder module into digital signals, eventually propagat to the host computer for analysis and processing, control commands can be manipulated to manipulate steering drilling servo mechanism, in order to achieve automatic control rotary steering drilling.

\section{CASE HISTORY: APPLICATION VERIFICATION AND GROUND SIMULATION RESULTS}

\subsection{Ground simulation computer HMI}

Shows the overall status of the system, can be set to synchronize the time to ensure on synchronization ground simulation computer and down-hole in realtime clock. Easy to storage and cleanup the historical data, remove historical data; Isoperimetric element of display operating temperature from down-hole BHA, rotating state, inclination, while the builtnegative mud pressure pulse control instructions.

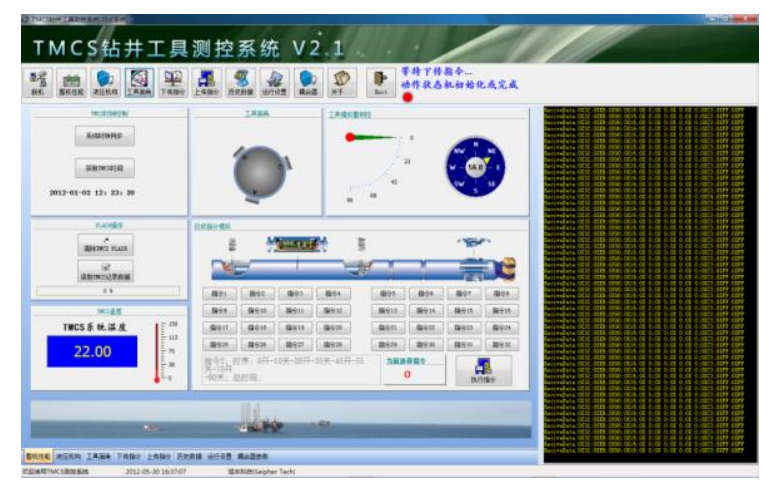

Figure 3. Ground simulation computer HMI 


\subsection{Inclination angle of the tool face and status display}

As shown below, displays various acquisition component of the primary and secondary angle sensor, the position of steering drilling servo mechanism of the rotary steering drilling tool solver, and tools tilted state while drilling with oblique azimuth data acquisition. Making visual display and recording the historical data. After contrastively and synthetically analysis on the ground simulation experiment data, inclination error $\leq \pm 0.1^{\circ}$, azimuth error $\leq \pm 2^{\circ}$, drift $\leq \pm 0.008^{\circ} /{ }^{\circ} \mathrm{C}$, verified effectively that steering drilling system measure the accuracy of data and analytic treatment, which get data from the sensor.

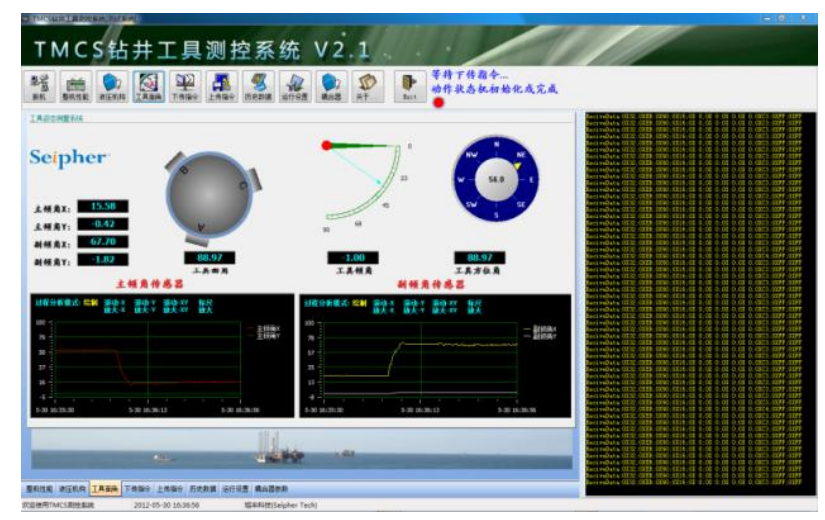

Figure 4. Ground simulation computer HMI - Display inclination, azimuth and tool face angle

\subsection{Servos status display and motion control}

Real-time acquisition and status solver position servo mechanism, display and control work status of micro brushless DC motor, work torque and speed, as well as control the supply of hydraulic fluid. Obtained from the ground experiment data analysis, measure the displacement range $0 \sim 50 \mathrm{~mm}$, and accuracy not less than $\pm 0.5 \mathrm{~mm}$.

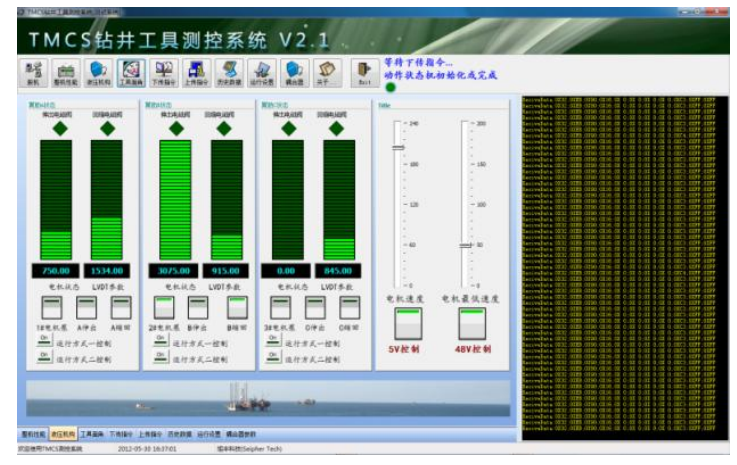

Figure 5. Ground simulation computer HMI - Servo mechanism status display and motion control

\section{CONCLUSIONS}

This system after completion of the ground simulation experiment, in June 2011, carried out a directional drilling experiment at an experimental base in Tianjin TangGu of China, it had drilling footage about $451 \mathrm{~m}$ from $601 \mathrm{~m} \sim 1062 \mathrm{~m}$, making maximum slope of $3.41\left(^{\circ}\right) / 30 \mathrm{~m}$. Test the rotary steering drilling tool to measure real-time acquisition with steering drilling, two-way communication, sent and received commands as to as to code and decode. The experiment results showed that there was real and credible results from ground simulation experiment, TMCS simulator completed in simulate down-hole actual drilling.

\section{REFERENCES}

[1] JIANG Shiquan, LI Hangxing, FU Xinsheng. Development of eccentric-shifting-control rotary-steering drilling toll and key technonlogy. Oil DRILLING \& PRODUCTION TECHNOLOGY.

[2] BARR U D, CLEGG J M, RUSSELL M K. Steerable rotary drilling with an experimental system. SPE/IADC 29382.

[3] SANDRO Poli, FRANCO Donat, JOACHIM Oppelt, etal. Advanced tools for advanced well: roatary closed loop drilling system. SPE 36884 